\title{
Normative Study to Correlate the Effects of Morphological Variables on Macular and Choroidal Thickness Using SD-OCT
}

\author{
Sumeet Chopra ${ }^{1}$, Preet Kanwar Singh Sodhi ${ }^{2}{ }^{*}$, Akash Deep Goel ${ }^{3}$, Sehajpreet Kaur ${ }^{1}$, \\ Gurkirat Singh Bajwa ${ }^{1}$ \\ ${ }^{1}$ Department of Ophthalmology, Dayanand Medical College and Hospital, Baba Farid University of Health Sciences, Ludhiana, India \\ ${ }^{2}$ Department of Vitreo-Retina, Sodhi Eye Hospital, Patiala, India \\ ${ }^{3}$ Department of Cornea, LV Prasad Eye Institute, Hyderabad, India
}

Email address:

choprasumeet36@gmail.com (S. Chopra),psodhi56@yahoo.co.in (P. K. S. Sodhi), akashdeep.goel@gmail.com (A. D Goel), sehaj2605@gmail.com (S. Kaur), gbajwa9@rediffmail.com (G. S. Bajwa)

${ }^{*}$ Corresponding author

\section{To cite this article:}

Sumeet Chopra, Preet Kanwar Singh Sodhi, Akashdeep Goel, Sehajpreet Kaur, Gurkirat Singh Bajwa. Normative Study to Correlate the Effects of Morphological Variables on Macular and Choroidal Thickness Using SD-OCT. International Journal of Ophthalmology \& Visual Science. Vol. 4, No. 1, 2019, pp. 1-6. doi: 10.11648/j.ijovs.20190401.11

Received: January 2, 2019; Accepted: January 21, 2019; Published: February 13, 2019

\begin{abstract}
To determine macular and choroidal thickness in healthy north Indian eyes and determine its variation with age and height using SD-OCT. The macula of 400 randomly selected healthy subjects (800 eyes) with no retinal and choroidal pathology was imaged with Cirrus SD-OCT. Macular thickness from all 9 regions of Early Treatment Diabetic Retinopathy Study was evaluated. Choroid was visualized by enhanced depth imaging technique. Choroidal thickness was measured sub foveally and at 500 microns intervals upto 1500 microns temporal and nasal to the fovea. The mean age of the subjects was 33.60 years \pm 14.033 and mean height was $163.5 \mathrm{cms} \pm 11.715$. Mean central macular thickness (CMT) was $242.59 \mu \mathrm{m} \pm$ 16.802 and mean sub foveal choroidal thickness was $325.18 \mu \mathrm{m} \pm 47.087$. CMT correlated significantly (Pearson's correlation coefficient) with age $(\mathrm{r}=0.120, \mathrm{p}<0.001)$ and height $(\mathrm{r}=0.258, \mathrm{p}<0.001)$. Choroidal thickness decreased with age and the correlation was statistically significant. Choroidal thickness also decreased with height with a statistically significant correlation, but no specific pattern was found. This normative database of choroidal and macular thickness by OCT will serve as baseline for diagnosing retinal pathologies and help in future research.
\end{abstract}

Keywords: Choroidal Thickness, Macular Thickness, Normative Study, Spectral-Domain Optical Coherence Tomography (SD-OCT), North Indian

\section{Introduction}

Macular edema is a common cause of visual impairment, and the degree of macular thickening is significantly correlated with visual acuity. [1] The assessment of the macular region is an important parameter in the management of several ophthalmic conditions like diabetic retinopathy, age-related macular degeneration, central serous chorio-retinopathy (CSCR), retinal vein occlusion etc where an increase in retinal thickness can be seen due to fluid accumulation.

Choroidal abnormalities such as vascular hyper permeability or loss and thinning are critical to the onset and progression of many chorioretinal diseases such as central serous chorioretinopathy, Vogt-Koyanagi-Harada disease, high myopia-related chorioretinal atrophies, age-related macular degeneration and polypoidal choroidal vasculopathy. The choroidal thickness (CT) may be affected by many local and systemic conditions.

With the recent development of enhanced depth imaging (EDI), in-vivo assessment of choroid has become an area of interest. EDI helps in better visualization of choroid and the chorio-scleral junction, which allows an accurate quantitative assessment of the choroid, which was not possible before. [2] 
Studies have reported significant differences in macular thickness amongst subjects of different race, gender and age. $[3,4]$ Thus it is desirable that measurements derived from the normative population be as close as possible to the population for which the instrument is to be used.

Recent literature has shown the effect of age, sex, axial length (AXL), refractive error, and diurnal variation on the Choroidal Thickness. [5, 6] Various studies have reported a normal range of choroidal thickness. [7-9] Previous reports on CT are mostly from the western world and from the Asian countries including Japan and China. Hence, we undertook this prospective study for evaluating the normal retinal and choroidal thickness in normal healthy north Indian subjects and also evaluated the effect of various morphological variables on the thicknesses.

\section{Materials and Methods}

This prospective observational study consisted of 400 randomly selected subjects (800 eyes), who visited the OPD, at Department of Ophthalmology, Dayanand Medical College \& Hospital, Ludhiana, Punjab, India in whom the chorioscleral junction could be identified with an image quality of $\geq$ 6/10. Prior approval from the Institutional Review Board of the institute was taken and informed consent was obtained from each subject. This study was conducted in accordance with the tenets of the Declaration of Helsinki for research involving human subjects. All subjects had a best corrected visual acuity of 20/20. Patients with any retinal or choroidal pathology, Refractive error of $> \pm 6$ Diopters sphere or $> \pm 3$ diopters cylinder, media opacities, intra ocular pressure $>21$ $\mathrm{mm}$, history of use of any drug causing retinopathy, history of any systemic disease with known ocular involvement, pregnant females, history of use of oral contraceptive pills, glaucoma were excluded from study.

Both eyes per subject were scanned. All scans had an image quality factor of $6 / 10$ or greater and were taken as close to fovea as possible, such that the thinnest point of the macula was imaged. OCT scanning was performed using CIRRUS HD-OCT (MODEL 5000, SD-OCT, CARL ZEISS MEDITEC, INC, DUBLIN, USA) with software version 6.5.

The fast macula thickness map was used, which comprises 3 concentric circles centred at the fovea that divided the macula into 3 zones; the fovea ( $1 \mathrm{~mm}$ diameter), the inner macula (1 to $3 \mathrm{~mm}$ ) and the outer macula (3 to $6 \mathrm{~mm}$ ). The 3 $\mathrm{mm}$ inner ring and $6 \mathrm{~mm}$ outer ring are further divided into four equal regions. The choroid was visualized by enhanced depth imaging (EDI) technique with spectral domain optical coherence tomography using a standardized scanning protocol. A single line of $6 \mathrm{~mm}$ length centered horizontally on the fovea was used for the visualization of the choroid. The vertical distance between the posterior edge of the hyper reflective RPE layer and the chorio-scleral interface was measured manually using the software calipers. Choroidal thickness was measured sub foveally and 500 microns intervals up to 1500 microns temporal and nasal to the fovea.

All the data including OCT parameters were stored in a MS Excel 2007 spreadsheet. Statistical analysis was done using SPSS software version 18.0.

\section{Results}

We included 800 eyes of 400 healthy subjects for analysis in this study. There were 206 men (412 eyes) and 194 women (388 eyes). Mean age of the subjects was 33.60 years \pm 14.033 and mean height was $163.5 \mathrm{~cm} \pm 11.715$. All patients were phakic.

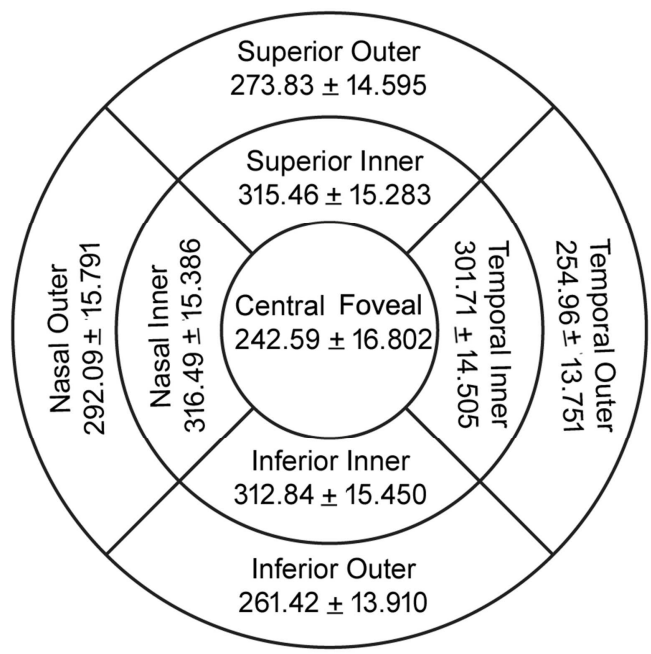

Figure 1. Normative Data for Retinal Thickness in Various ETDRS Sub Fields.

Mean Foveal thickness was $242.59 \mu \mathrm{m} \pm 16.802$. The inner circle of ETDRS grid showed maximum retinal thickness and there was a decrease towards periphery. The retina was the thickest on nasal side with maximum thickness of inner nasal macula (Figure 1).

Table 1. Normative Data for Choroidal Thickness.

\begin{tabular}{ll}
\hline Area of Measurement & Mean Choroidal Thickness $(\boldsymbol{\mu m}) \pm$ Standard Deviation \\
\hline Temporal $1500 \mu \mathrm{m}$ & $293.40 \pm 46.686$ \\
Temporal $1000 \mu \mathrm{m}$ & $306.59 \pm 46.358$ \\
Temporal $500 \mu \mathrm{m}$ & $316.32 \pm 46.723$ \\
Sub Foveal & $325.18 \pm 47.087$ \\
Nasal $500 \mu \mathrm{m}$ & $314.42 \pm 47.889$ \\
Nasal $1000 \mu \mathrm{m}$ & $302.81 \pm 49.649$ \\
Nasal $1500 \mu \mathrm{m}$ & $286.09 \pm 52.013$ \\
\hline
\end{tabular}

Mean sub foveal choroidal thickness was $325.18 \mu \mathrm{m} \pm 47.087$. The choroid was thickest at subfoveal area. A gradual decrease of choroidal thickness was noted towards periphery. This decrease was more on nasal side than on the temporal side (Table 1). 
Table 2. Mean Retinal Thickness ( $\mu m$ ) Variations with Age \pm S.D.

\begin{tabular}{|c|c|c|c|c|c|c|c|c|}
\hline $\begin{array}{l}\text { ETDRS Sub } \\
\text { Field }\end{array}$ & $\begin{array}{l}\text { Group A } \\
\text { ( } \leq 20 \text { Years) } \\
\mathrm{N}=188 \\
\end{array}$ & $\begin{array}{l}\text { Group B } \\
\text { (21-30 Years) } \\
\mathrm{N}=178 \\
\end{array}$ & $\begin{array}{l}\text { Group C } \\
\text { (31-40 Years) } \\
\mathrm{N}=154 \\
\end{array}$ & $\begin{array}{l}\text { Group D } \\
(41-50 \text { Years) } \\
N=180 \\
\end{array}$ & $\begin{array}{l}\text { Group } \mathrm{E} \\
\text { (51-60 Years) } \\
\mathrm{N}=86\end{array}$ & $\begin{array}{l}\text { Group F } \\
\begin{array}{l}(>60 \text { Years) } \\
N=14\end{array}\end{array}$ & $\begin{array}{l}\text { Total } \\
\mathbf{N}=\mathbf{8 0 0}\end{array}$ & $\begin{array}{l}\text { Overall } \\
\text { p value }\end{array}$ \\
\hline Foveal & $240.53 \pm 15.657$ & $243.15 \pm 15.168$ & $241.76 \pm 20.315$ & $243.36 \pm 16.459$ & $244.69 \pm 16.674$ & $249.36 \pm 11.222$ & $242.59 \pm 16.802$ & 0.192 \\
\hline Temporal Inner & $300.65 \pm 14.416$ & $303.08 \pm 13.528$ & $300.74 \pm 14.379$ & $302.00 \pm 14.515$ & $301.03 \pm 17.352$ & $309.43 \pm 5.095$ & $301.71 \pm 14.505$ & 0.186 \\
\hline Superior Inner & $313.79 \pm 15.085$ & $316.89 \pm 14.637$ & $314.82 \pm 14.159$ & $315.63 \pm 17.143$ & $315.64 \pm 15.283$ & $323.36 \pm 9.516$ & $315.46 \pm 15.283$ & 0.164 \\
\hline Nasal Inner & $315.28 \pm 15.237$ & $317.42 \pm 15.056$ & $315.84 \pm 15.601$ & $316.83 \pm 15.850$ & $316.56 \pm 15.992$ & $323.43 \pm 5.971$ & $316.49 \pm 15.386$ & 0.413 \\
\hline Inferior Inner & $311.37 \pm 15.145$ & $314.16 \pm 15.217$ & $312.31 \pm 15.443$ & $312.53 \pm 16.328$ & $313.87 \pm 15.456$ & $319.29 \pm 8.260$ & $312.84 \pm 15.450$ & 0.299 \\
\hline Temporal Outer & $254.03 \pm 14.283$ & $255.01 \pm 12.999$ & $254.29 \pm 12.306$ & $255.97 \pm 15.447$ & $255.36 \pm 13.117$ & $258.50 \pm 12.113$ & $254.96 \pm 13.751$ & 0.671 \\
\hline Superior Outer & $272.06 \pm 14.671$ & $273.43 \pm 13.272$ & $274.19 \pm 15.110$ & $274.49 \pm 12.739$ & $276.00 \pm 19.094$ & $276.71 \pm 14.019$ & $273.83 \pm 14.595$ & 0.326 \\
\hline Nasal Outer & $290.93 \pm 15.875$ & $291.49 \pm 16.520$ & $292.29 \pm 13.528$ & $293.06 \pm 17.121$ & $292.84 \pm 14.939$ & $296.00 \pm 16.678$ & $292.09 \pm 15.791$ & 0.698 \\
\hline Inferior Outer & $260.50 \pm 13.041$ & $260.97 \pm 15.042$ & $261.93 \pm 12.697$ & $261.55 \pm 14.990$ & $262.87 \pm 13.281$ & $263.43 \pm 13.535$ & $261.42 \pm 13.910$ & 0.783 \\
\hline
\end{tabular}

Central foveal thickness was minimum in age $<20$ years and maximum in the older age group with age $>60$ years while it was almost similar in age groups from 21-60 years (Table 2). The central foveal thickness had a significant positive correlation with age $(\mathrm{r}=0.120, \mathrm{p}<.001)$ with an increase of $1.43 \mu \mathrm{m}$ thickness per decade. The retinal thickness in the superior outer quadrant had a statistically significant negative correlation with age with a decrease of $0.067 \mu \mathrm{m}$ per decade (Table 3 ).

Table 3. Retinal thickness correlation with age.

\begin{tabular}{llllll}
\hline $\begin{array}{l}\text { ETDRS Sub } \\
\text { Field }\end{array}$ & $\begin{array}{l}\text { Minimum } \\
\text { Thickness }(\boldsymbol{\mu m})\end{array}$ & $\begin{array}{l}\text { Maximum } \\
\text { Thickness }(\boldsymbol{\mu m})\end{array}$ & $\begin{array}{l}\text { Mean Thickness } \\
(\boldsymbol{\mu m}) \pm \text { S.D. }\end{array}$ & $\begin{array}{l}\text { Change per } \\
\text { Decade }(\boldsymbol{\mu m})\end{array}$ & $\begin{array}{l}\text { Significance } \\
(\mathbf{p}-\text { Value })\end{array}$ \\
\hline Central foveal & 190 & 305 & $242.59 \pm 16.802$ & 1.43 & 0.000 \\
Temporal Inner & 219 & 338 & $301.71 \pm 14.505$ & -0.18 & 0.120 \\
Superior Inner & 208 & 360 & $315.46 \pm 15.283$ & -0.4 & -0.017 \\
Nasal Inner & 267 & 357 & $316.49 \pm 15.386$ & 0.11 & -0.022 \\
Inferior Inner & 250 & 349 & $312.84 \pm 15.450$ & 0.22 & -0.010 \\
Temporal Outer & 222 & 357 & $254.96 \pm 13.751$ & 0.42 & 0.020 \\
Superior Outer & 241 & 395 & $273.83 \pm 14.595$ & -0.69 & 0.043 \\
Nasal Outer & 247 & 341 & $292.09 \pm 15.791$ & -0.43 & -0.067 \\
Inferior Outer & 205 & 321 & $261.42 \pm 13.910$ & -0.16 & -0.038 \\
\hline
\end{tabular}

Table 4. Mean Choroidal Thickness ( $\mu \mathrm{m})$ Variations with Age \pm S.D.

\begin{tabular}{|c|c|c|c|c|c|c|c|c|}
\hline $\begin{array}{l}\text { Area of } \\
\text { Measurement }\end{array}$ & $\begin{array}{l}\text { Group A } \\
(\leq 20 \text { Years) } \\
\mathrm{N}=188\end{array}$ & $\begin{array}{l}\text { Group B } \\
\text { (21-30 Years) } \\
\mathrm{N}=178 \\
\end{array}$ & $\begin{array}{l}\text { Group C } \\
\text { (31-40 Years) } \\
N=154\end{array}$ & $\begin{array}{l}\text { Group D } \\
\text { (41-50 Years) } \\
N=180\end{array}$ & $\begin{array}{l}\text { Group E } \\
\text { (51-60 Years) } \\
\mathrm{N}=86\end{array}$ & $\begin{array}{l}\text { Group } F \\
(>60 \text { Years) } \\
N=14\end{array}$ & $\begin{array}{l}\text { Total } \\
\mathbf{N}=\mathbf{8 0 0}\end{array}$ & $\begin{array}{l}\text { Over all } \\
\text { p value }\end{array}$ \\
\hline Temporal $1500 \mu \mathrm{m}$ & $292.98 \pm 48.724$ & $291.26 \pm 49.012$ & $293.94 \pm 46.666$ & $294.75 \pm 43.600$ & $296.17 \pm 44.318$ & $285.93 \pm 47.433$ & $293.40 \pm 46.686$ & 0.943 \\
\hline Temporal $1000 \mu \mathrm{m}$ & $305.86 \pm 47.940$ & $305.06 \pm 47.837$ & $305.76 \pm 47.990$ & $308.91 \pm 43.995$ & $308.99 \pm 43.104$ & $300.29 \pm 41.926$ & $306.59 \pm 46.358$ & 0.943 \\
\hline Temporal $500 \mu \mathrm{m}$ & $315.70 \pm 48.061$ & $314.78 \pm 47.671$ & $315.51 \pm 47.991$ & $318.48 \pm 45.445$ & $318.65 \pm 42.998$ & $310.93 \pm 46.861$ & $316.32 \pm 46.723$ & 0.958 \\
\hline Sub Foveal & $324.25 \pm 48.058$ & $324.26 \pm 47.239$ & $324.31 \pm 48.508$ & $327.78 \pm 45.843$ & $326.34 \pm 45.144$ & $318.29 \pm 50.237$ & $325.18 \pm 47.087$ & 0.955 \\
\hline Nasal $500 \mu \mathrm{m}$ & $313.46 \pm 48.067$ & $313.44 \pm 48.903$ & $314.45 \pm 48.214$ & $316.07 \pm 47.206$ & $316.17 \pm 47.043$ & $307.36 \pm 50.066$ & $314.42 \pm 47.889$ & 0.978 \\
\hline Nasal $1000 \mu \mathrm{m}$ & $302.81 \pm 48.538$ & $302.03 \pm 51.200$ & $302.62 \pm 49.307$ & $302.61 \pm 49.896$ & $306.31 \pm 50.043$ & $295.86 \pm 50.118$ & $302.81 \pm 49.649$ & 0.980 \\
\hline Nasal $1500 \mu \mathrm{m}$ & $287.84 \pm 49.167$ & $284.43 \pm 55.195$ & $286.33 \pm 52.495$ & $284.69 \pm 51.630$ & $289.77 \pm 52.826$ & $276.50 \pm 48.381$ & $286.09 \pm 52.013$ & 0.921 \\
\hline
\end{tabular}

Choroidal thickness was the least in the individuals with age $>60$ years (Table 4 ). The subfoveal choroidal thickness had a significant negative correlation with age $(\mathrm{r}=-0.297, \mathrm{p}<.001)$ with a decrease of $9.97 \mu \mathrm{m}$ per decade (Table 5).

Table 5. Choroidal thickness correlation with age.

\begin{tabular}{llllll}
\hline $\begin{array}{l}\text { Area of } \\
\text { Measurement }\end{array}$ & $\begin{array}{l}\text { Minimum } \\
\text { Thickness }(\boldsymbol{\mu m})\end{array}$ & $\begin{array}{l}\text { Maximum } \\
\text { Thickness }(\boldsymbol{\mu m})\end{array}$ & $\begin{array}{l}\text { Mean Thickness } \\
(\boldsymbol{\mu m}) \pm \text { S.D. }\end{array}$ & $\begin{array}{l}\text { Change per } \\
\text { Decade }(\boldsymbol{\mu m})\end{array}$ & $\begin{array}{l}\text { Correlation }(\mathbf{r}) \\
\text { Significance }(\mathbf{p}-\text { Value) }\end{array}$ \\
\hline Temporal $1500 \mu \mathrm{m}$ & 134 & 443 & $293.40 \pm 46.686$ & -10.29 & -0.309 \\
Temporal $1000 \mu \mathrm{m}$ & 144 & 436 & $306.59 \pm 46.358$ & -9.95 & -0.301 \\
Temporal $500 \mu \mathrm{m}$ & 154 & 439 & $316.32 \pm 46.723$ & -10.19 & -0.306 \\
Sub Foveal & 157 & 436 & $325.18 \pm 47.087$ & -9.97 & -0.297 \\
Nasal $500 \mu \mathrm{m}$ & 160 & 448 & $314.42 \pm 47.889$ & -9.14 & -0.268 \\
Nasal $1000 \mu \mathrm{m}$ & 141 & 438 & $302.81 \pm 49.649$ & -7.97 & -0.225 \\
Nasal $1500 \mu \mathrm{m}$ & 112 & 413 & $286.09 \pm 52.013$ & -7.01 & -0.000 \\
\hline
\end{tabular}


Table 6. Mean Retinal Thickness ( $\mu \mathrm{m})$ Variations with Height \pm S.D.

\begin{tabular}{|c|c|c|c|c|c|}
\hline \multirow{2}{*}{ ETDRS Sub Field } & \multirow{2}{*}{$\begin{array}{l}\text { Group X } \\
(\leq 150 \mathrm{Cms}) \mathrm{N}=110\end{array}$} & \multirow{2}{*}{$\begin{array}{l}\text { Group Y } \\
(151-165 \mathrm{Cms}) \mathrm{N}=352\end{array}$} & \multirow{2}{*}{$\begin{array}{l}\text { Group Z } \\
(>165 \mathrm{Cms}) \mathrm{N}=338\end{array}$} & \multirow{2}{*}{$\begin{array}{l}\text { Total } \\
\mathbf{N}=\mathbf{8 0 0}\end{array}$} & \multirow{2}{*}{ Over all p value } \\
\hline & & & & & \\
\hline Foveal & $238.95 \pm 16.193$ & $238.69 \pm 16.427$ & $247.83 \pm 15.992$ & $242.59 \pm 16.802$ & $<0.001$ \\
\hline Temporal Inner & $298.50 \pm 10.982$ & $297.84 \pm 14.065$ & $306.78 \pm 14.459$ & $301.71 \pm 14.505$ & $<0.001$ \\
\hline Superior Inner & $311.69 \pm 10.596$ & $312.11 \pm 14.837$ & $320.17 \pm 15.798$ & $315.46 \pm 15.283$ & $<0.001$ \\
\hline Nasal Inner & $313.00 \pm 10.868$ & $312.87 \pm 15.540$ & $321.40 \pm 15.156$ & $316.49 \pm 15.386$ & $<0.001$ \\
\hline Inferior Inner & $309.68 \pm 11.298$ & $309.25 \pm 15.172$ & $317.61 \pm 15.664$ & $312.84 \pm 15.450$ & $<0.001$ \\
\hline Temporal Outer & $250.99 \pm 9.613$ & $252.49 \pm 14.626$ & $258.82 \pm 13.006$ & $254.96 \pm 13.751$ & $<0.001$ \\
\hline Superior Outer & $270.95 \pm 10.866$ & $273.44 \pm 16.985$ & $275.16 \pm 12.716$ & $273.83 \pm 14.595$ & 0.025 \\
\hline Nasal Outer & $289.98 \pm 12.022$ & $291.04 \pm 16.121$ & $293.86 \pm 16.372$ & $292.09 \pm 15.791$ & 0.020 \\
\hline Inferior Outer & $259.40 \pm 12.302$ & $259.80 \pm 14.568$ & $263.77 \pm 13.383$ & $261.42 \pm 13.910$ & $<0.001$ \\
\hline
\end{tabular}

Retinal thickness was the maximum in group- $Z$ i.e. with a height more than $165 \mathrm{cms}$, while it was almost similar in other height groups (Table 6). The retinal thickness had a significant positive correlation with height with a variation in the range of 2.250-5.550 $\mu \mathrm{m}$ in the each height group in various ETDRS sub fields (Table 7).

Table 7. Retinal thickness correlation with height.

\begin{tabular}{llllll}
\hline ETDRS Sub Field & $\begin{array}{l}\text { Minimum } \\
\text { Thickness }(\boldsymbol{\mu m})\end{array}$ & $\begin{array}{l}\text { Maximum } \\
\text { Thickness }(\boldsymbol{\mu m})\end{array}$ & $\begin{array}{l}\text { Mean Thickness } \\
(\boldsymbol{\mu m}) \pm \text { S.D. }\end{array}$ & $\begin{array}{l}\text { Change per } \\
\text { Group }(\boldsymbol{\mu m})\end{array}$ & $\begin{array}{l}\text { Correlation }(\mathbf{r}) \\
(\mathbf{p}-\text { Value })\end{array}$ \\
\hline Central foveal & 190 & 305 & $242.59 \pm 16.802$ & -5.55 & 0.258 \\
Temporal Inner & 219 & 338 & $301.71 \pm 14.505$ & -5.34 & 0.000 \\
Superior Inner & 208 & 360 & $315.46 \pm 15.283$ & -5.115 & 0.288 \\
Nasal Inner & 267 & 357 & $316.49 \pm 15.386$ & -5.415 & 0.261 \\
Inferior Inner & 250 & 349 & $312.84 \pm 15.450$ & -5.295 & 0.275 \\
Temporal Outer & 222 & 357 & $254.96 \pm 13.751$ & -4.32 & 0.268 \\
Superior Outer & 241 & 395 & $273.83 \pm 14.595$ & -2.25 & 0.245 \\
Nasal Outer & 247 & 341 & $292.09 \pm 15.791$ & -2.58 & 0.000 \\
Inferior Outer & 205 & 321 & $261.42 \pm 13.910$ & -2.685 & 0.000 \\
\hline
\end{tabular}

Table 8. Mean Choroidal Thickness ( $\mu \mathrm{m})$ Variations with Height \pm S.D.

\begin{tabular}{|c|c|c|c|c|c|}
\hline \multirow{2}{*}{ Area of Measurement } & \multirow{2}{*}{$\begin{array}{l}\text { Group X } \\
(\leq 150 \mathrm{Cms}) \mathrm{N}=110\end{array}$} & \multirow{2}{*}{$\begin{array}{l}\text { Group Y } \\
(151-165 \text { Cms }) ~ N=352\end{array}$} & \multirow{2}{*}{$\begin{array}{l}\text { Group Z } \\
(>165 \mathrm{Cms}) \mathrm{N}=338\end{array}$} & \multirow{2}{*}{$\begin{array}{l}\text { Total } \\
\mathbf{N}=\mathbf{8 0 0}\end{array}$} & \multirow{2}{*}{ Over all p value } \\
\hline & & & & & \\
\hline Temporal $1500 \mu \mathrm{m}$ & $288.96 \pm 46.586$ & $297.00 \pm 47.269$ & $291.09 \pm 45.969$ & $293.40 \pm 46.686$ & 0.142 \\
\hline Temporal $1000 \mu \mathrm{m}$ & $304.77 \pm 48.705$ & $309.97 \pm 45.412$ & $303.66 \pm 46.458$ & $306.59 \pm 46.358$ & 0.184 \\
\hline Temporal $500 \mu \mathrm{m}$ & $313.17 \pm 48.646$ & $319.97 \pm 45.231$ & $313.53 \pm 47.482$ & $316.32 \pm 46.723$ & 0.145 \\
\hline Sub Foveal & $321.93 \pm 49.868$ & $328.80 \pm 45.171$ & $322.47 \pm 47.987$ & $325.18 \pm 47.087$ & 0.155 \\
\hline Nasal $500 \mu \mathrm{m}$ & $310.25 \pm 50.341$ & $317.54 \pm 46.269$ & $312.53 \pm 48.667$ & $314.42 \pm 47.889$ & 0.241 \\
\hline Nasal $1000 \mu \mathrm{m}$ & $298.67 \pm 50.955$ & $305.79 \pm 48.607$ & $301.05 \pm 50.258$ & $302.81 \pm 49.649$ & 0.294 \\
\hline Nasal $1500 \mu \mathrm{m}$ & $281.59 \pm 52.693$ & $288.95 \pm 51.212$ & $284.58 \pm 52.602$ & $286.09 \pm 52.013$ & 0.337 \\
\hline
\end{tabular}

The maximum choroidal thickness was observed in Group-Y i.e. with height range from 151-165 cms and it showed a fall in other groups that is with higher and lower height groups (Table 8). The choroidal thickness had a significant negative correlation with height with a decrease in the range of 4.020 to $5.580 \mu \mathrm{m}$ at various points of measurements (Table 9).

Table 9. Choroidal thickness correlation with height.

\begin{tabular}{|c|c|c|c|c|c|c|}
\hline Area of Measurement & $\begin{array}{l}\text { Minimum } \\
\text { Thickness }(\mu \mathrm{m})\end{array}$ & $\begin{array}{l}\text { Maximum } \\
\text { Thickness }(\mu \mathrm{m})\end{array}$ & $\begin{array}{l}\text { Mean Thickness } \\
(\mu \mathrm{m}) \pm \text { S.D. }\end{array}$ & $\begin{array}{l}\text { Change per group } \\
(\mu \mathrm{m})\end{array}$ & $\begin{array}{l}\text { Correlation } \\
\text { (r) }\end{array}$ & $\begin{array}{l}\text { Significance (p- } \\
\text { Value) }\end{array}$ \\
\hline Temporal $1500 \mu \mathrm{m}$ & 134 & 443 & $293.40 \pm 46.686$ & 4.020 & -0.067 & 0.029 \\
\hline Temporal $1000 \mu \mathrm{m}$ & 144 & 436 & $306.59 \pm 46.358$ & 5.580 & -0.094 & 0.004 \\
\hline Temporal $500 \mu \mathrm{m}$ & 154 & 439 & $316.32 \pm 46.723$ & 5.385 & -0.090 & 0.005 \\
\hline Sub Foveal & 157 & 436 & $325.18 \pm 47.087$ & 5.445 & -0.090 & 0.005 \\
\hline Nasal $500 \mu \mathrm{m}$ & 160 & 448 & $314.42 \pm 47.889$ & 4.785 & -0.078 & 0.014 \\
\hline Nasal $1000 \mu \mathrm{m}$ & 141 & 438 & $302.81 \pm 49.649$ & 4.980 & -0.078 & 0.013 \\
\hline Nasal $1500 \mu \mathrm{m}$ & 112 & 413 & $286.09 \pm 52.013$ & 4.335 & -0.065 & 0.033 \\
\hline
\end{tabular}




\section{Discussion}

The assessment of the macular and choroidal region is an important parameter in the management of several ophthalmic diseases. Prior to labeling the macula or choroid as abnormal, it is important to determine the range of normal macular and choroidal thickness and the factors on which they depend.

Our study consisted of 487 subjects initially out of which those 400 were included in whom the chorio-scleral junction could be identified with an image quality of $\geq 6 / 10$. Out of the 400 included patients $206(51.50 \%)$ were males and 194 $(48.50 \%)$ were females. All the patients were phakic and both eyes were evaluated. The mean age of the subjects was $33.60 \pm 14.033$ years (range 9-67 years).

The mean foveal thickness was $242.59 \mu \mathrm{m} \pm 16.802$. Previous studies have shown that Asians and AfricanAmericans have thinner maculae when compared to Caucasians. $[10,11]$ The mean thickness in the central $1 \mathrm{~mm}$ diameter area was found to be $176.4 \mu \mathrm{m}$ in Chinese, [12] $181.2 \pm 18.4 \mu \mathrm{m}$ in Indians, [13] $183.2 \pm 1.3 \mu \mathrm{m}$ in Thai, [14] $209.5 \pm 26.7 \mu \mathrm{m}$ in Japanese, [15] and $212 \pm 20 \mu \mathrm{m}$ in Caucasians [16] using different OCT machines. Our study showed almost similar retinal thickness with the studies done by Song et al. [17], Gupta et al. [18] using Cirrus HD-OCT. The difference in the measurements is due to the fact that time domain (TD-OCT) measures retinal thickness as the distance between internal limiting membrane (ILM) and the third hyper-reflective band, whereas SD OCT measures the distance between ILM and the retinal pigment epithelium (RPE) resulting in higher SD OCT readings compared to those obtained by TD-OCT.

On evaluating the macular thickness in the 9 ETDRS regions in our population, the nasal macula (inner and outer areas) was significantly thicker than the temporal macula, a finding consistent with previous studies. [4, 18, 20] This could be because of the thicker papillo-macular bundle of the retinal nerve fiber layer (RNFL) in this region.

We found no specific pattern of retinal variation with height, although a positive statistically significant correlation was found at all the measured points $(\mathrm{p}<.001)$. A positive and significant correlation between the retinal thickness and body height has been reported by Takehiro Yamashita et al. [20]

Using the criteria of mean \pm 2 Standard Deviations, which includes $95 \%$ of the population, we suggest that $210-275 \mu \mathrm{m}$ be taken as the normal range for central foveal thickness in the north Indian population using CIRRUS OCT.

Our study of a healthy north Indian population showed a mean subfoveal choroidal thickness of $325.18 \pm 47.087 \mu \mathrm{m}$ with a mean age of 33.60 years, which was more than a Korean population [21] $(307.3 \pm 95.2 \mu \mathrm{m})$ with a mean age of 40.18 years, an adult Spanish population [22] (305.6 \pm 102.6 $\mu \mathrm{m})$ with a mean age of 53 years and a Chinese population [23] $(261.9 \pm 88.4 \mu \mathrm{m})$ with a mean age of 49.73 years and lesser than a Japanese population [24] $(354 \pm 111 \mu \mathrm{m})$ with a mean age of $39.4 \pm 16.0$, a mixed ethnic population of UK [25] $(332 \pm 90 \mu \mathrm{m})$ with a mean age of $24.46 \pm 1.12$ and a Turkish population [26] in which the mean subfoveal CT was $348 \pm$ $68 \mu \mathrm{m}$. The differences in mean subfoveal choroidal thickness may result from differences in the mean age of each study.

Choroidal thickness was thickest below the fovea. The present study also showed that the nasal choroid was significantly thinner than the subfoveal and temporal choroid. The reason for could be the choroidal watershed, which isolates the choroidal circulation, as seen on fluorescein or indocyanine green angiography.

Choroidal thickness was strongly negatively correlated with age at all the measured locations suggesting that progressive choroidal thinning occurs over time in normal eyes. However, our study did not have the power to show a statistically significant difference between younger subjects and subjects older than 60 years of age due to small number of subjects older than 60 years with good quality images.

We found no specific pattern of choroidal variation with height, although a small negative statistically significant correlation was found below the fovea $(r=-.090 \mathrm{p}=.005)$, so its clinical significance cannot be commented on.

According to our knowledge, this is the first study which describes normative values in healthy North Indian subjects. Intra-observer and Intra-observer variations could alter the results, these could not be evaluated in the study done. Intraeye variations were evaluated though, which didn't show any statistically significant co-relation.

\section{Conclusions}

A normative database was established for the north Indian population of the retinal and choroidal thickness which is important to diagnose the abnormalities. Normal central retinal thickness for a north Indian population is $242.59 \mu \mathrm{m} \pm$ 16.802 and subfoveal choroidal thickness is $325.18 \mu \mathrm{m} \pm$ 47.087 using Cirrus HD-OCT. Central macular thickness is positively correlated $(\mathrm{r}=0.120, \mathrm{p}<.001)$ while sub foveal choroidal thickness is negatively correlated $(\mathrm{r}=-0.297$, $\mathrm{p}$ $<.001$ ) with age. Central macular thickness is positively correlated $(\mathrm{r}=.0258, \mathrm{p}<.001)$ while sub foveal choroidal thickness is negatively correlated $(\mathrm{r}=-0.090, \mathrm{p}=.005)$ with height.

\section{Acknowledgements}

The authors acknowledge the role of the entire staff of Department of Ophthalmology, Dayanand Medical College and Hospital, Ludhiana, Punjab where the study was conducted. The management's role for the conduct of study is also acknowledged. The role of Dr Sanjeev Maheshwari for biostatics and preparation of the manuscript is also deeply acknowledged. 


\section{References}

[1] Nussenblatt RB, Kaufman SC, Palestine AG, Davis MD, Ferris FL Macular thickening and visual acuity: Measurements in patients with cystoid macular edema. Ophthalmology. 1987; 94:1134-1139.

[2] Spaide RF, Koizumi H, Pozonni MC. Enhanced depth imaging spectral-domain optical coherence tomography. Am J Ophthalmol. 2008; 146 (4): 496-500.

[3] Choovuthayakorn J, Watanachai N, Chaikitmongkol V, Patikulsila D, Kunavisarut P, Ittipunkul N: Macular thickness measured by spectral-domain optical coherence tomography in healthy Thai eyes. Jpn J Ophthalmol. 2012; 56 (6):569-76.

[4] Zia S Pradhan, Andrew Braganza, Lekha M Abraham: Determinants of macular thickness in normal Indian eyes. J. Cli Oph. Res. 2013; 1 (1):11-16.

[5] Barteselli G, Chhablani J, El-Emam S, Wang H, Chuang J, Kozak I, et al. Choroidal volume variations with age, axial length, and sex in healthy subjects: A three dimensional analysis. Ophthalmology 2012; 119:2572-8.

[6] Tan CS, Ouyang Y, Ruiz H, Sadda SR. Diurnal variation of choroidal thickness in normal, healthy subjects measured by spectral domain optical coherence tomography. Invest Ophthalmol Vis Sci. 2012; 53:261-6.

[7] Manjunath V, Taha M, Fujimoto JG, Duker JS. Choroidal thickness in normal eyes measured using Cirrus HD optical coherence tomography. Am J Ophthalmol. 2010; 150 (3):3259.

[8] Hirata M, Tsujikawa A, Matsumoto A, Hangai M, Ooto S, Yamashiro K, et al. Macular choroidal thickness and volume in normal subjects measured by swept source optical coherence tomography. Invest Ophthal Vis Sci. 2011; 52:4971-8.

[9] Ikuno Y, Kawaguchi K, Nouchi T, Yasuno Y. Choroidal Thickness in healthy Japanese subjects. Invt Ophthalmol Vis Sci. 2010; 51 (4):2174-6.

[10] Kelty PJ, Payne JF, Trivedi RH, Kelty J, Bowie EM, Burger BM. Macular thickness assessment in healthy eyes based on ethnicity using Stratus OCT optical coherence tomography. Invest Ophthalmol Vis Sci. 2008; 49 (6):2668-72.

[11] Huang J, Liu X, Wu Z, Xiao H, Dustin L, Sadda S. Macular thickness measurements in normal eyes with time-domain and Fourier domain optical coherence tomography. Retina 2009; 29 (7):980-7.

[12] Duan X. R., Liang Y. B., Friedman D. S., et al. Normal macular thickness measurements using optical coherence tomography in healthy eyes of adult Chinese persons. Ophthalmology. 2010; 117 (8):1585-1594.

[13] Tewari H, Wagh V, Sony P, Venkatesh P, Singh R. Macular thickness evaluation using the optical coherence tomography in normal Indian eyes. Indian J Ophthalmol. 2004; 52 (3):199204.
[14] Manassakorn A, Chaidaroon W, Ausayakhun S, Aupapong S, Wattananikorn S. Normative database of retinal nerve fiber layer and macular retinal thickness in a Thai population. Jpn J Ophthalmol. 2008; 52 (6):450-6.

[15] Oshitari T, Hanawa K, Adachi-Usami E. Macular and retinal nerve fiber layer thickness in Japanese measured by Stratus optical coherence tomography. Clin Ophthalmol. 2007; 1:13340.

[16] Chan A, Duker JS, Ko TH, Fujimoto JG, Schuman JS. Normal macular thickness measurements in healthy eyes using Stratus optical coherence tomography. Arch Ophthalmol. 2006; 124 (2):193-8.

[17] Song WK, Lee SC, Lee ES, Kim CY, Kim SS. Macular Thickness Variations with Sex, Age, and Axial Length in Healthy Subjects: A Spectral Domain-OCT Study. Invest Ophthalmol Vis Sci. 2010; 51 (8):3913-8.

[18] Gupta P, Sidhartha E, Tham YC, Chua DK, Liao J, Cheng CY, et al. Determinants of macular thickness using spectral domain optical coherence tomography in healthy eyes: the Singapore Chinese Eye study. Invest Ophthalmol Vis Sci. 2013; 54:7968-76.

[19] Bindu Appukuttan, Anantharaman Giridhar, Mahesh Gopalakrishnan, and Sobha Sivaprasad. Normative spectral domain optical coherence tomography data on macular and retinal nerve fiber layer thickness in Indians. Indian J Ophthalmol. 2014 Mar; 62 (3): 316-321.

[20] Terasaki H, Shirasawa M, Yamashita T, et al. Comparison of foveal microstructure imaging with different spectral domain optical coherence tomography machines. Ophthalmology. 2012; 119 (11):2319-27.

[21] Kim M, Kim SS, Koh HJ, Lee SC. Choroidal thickness, age and refractive error in healthy Korean subjects. Optom Vis Sci. 2014; 91:491-496.

[22] Ruiz-Moreno JM, Flores-Moreno I, Lugo F, Ruiz-Medrano J, Montero JA, Akiba M. Macular choroidal thickness in normal pediatric population measured by swept-source optical coherence tomography. Invest Ophthalmol Vis Sci. 2013; 54:353-359.

[23] Xiaoyan Ding, Jiaqing Li, Jing Zeng, Wei Ma, Ran Liu, Tao Li, Shanshan Yu, and Shibo Tang. Choroidal Thickness in Healthy Chinese Subjects. IOVS, December 2011; Vol. 52, No. 13:9555-9560.

[24] Yasushi Ikuno, Kana Kawaguchi, Takeyoshi Nouchi,, and Yoshiaki Yasuno Choroidal Thickness in Healthy Japanese Subjects. Investigative Ophthalmology \& Visual Science, April 2010; Vol. 51, No. 4: 2174-2176.

[25] Rahman W, Chen FK, Yeoh J, Patel P, Tufail A, Da Cruz L. Repeatability of manual subfoveal choroidal thickness measurements in healthy subjects using the technique of enhanced depth imaging optical coherence tomography. Invest Ophthalmol Vis Sci 2011; 52:2267-71.

[26] Ihsan Yilmaz, Abdullah O, Murat K, Sibel A, Hande M. O, Dilek Y, Alper A, Ahmet T. Y, Ahmet D. Correlation of choroidal thickness and Body Mass Index. Retina 35:20852090, 2015. 